\title{
Auriculibuller fuscus gen. nov., sp. nov. and Bullera japonica sp. nov., novel taxa in the Tremellales
}

Correspondence José Paulo Sampaio jss@fct.unl.pt

\author{
José Paulo Sampaio, ${ }^{1}$ João Inácio, ${ }^{1}$ Álvaro Fonseca, ${ }^{1}$ Mário Gadanho, ${ }^{1}$ \\ Isabel Spencer-Martins, ${ }^{1}$ Gloria Scorzetti ${ }^{2}$ and Jack W. Fell ${ }^{2}$ \\ ${ }^{1}$ Centro de Recursos Microbiológicos, Secção Autónoma de Biotecnologia, Faculdade de \\ Ciências e Tecnologia, Universidade Nova de Lisboa, 2829-516 Caparica, Portugal \\ ${ }^{2}$ Rosenstiel School of Marine and Atmospheric Science, University of Miami, \\ 4600 Rickenbacker Causeway, Key Biscayne, FL 33419, USA
}

\section{INTRODUCTION}

The phenotypic circumscription of the basidiomycetous anamorphic genus Bullera Derx is based on the production of rotationally symmetrical ballistoconidia, absence of bud-forming stalks, white to cream or yellowish cultures, inositol assimilation, production of starch-like compounds (a few exceptions are known for the two latter tests), CoQ10 as the major ubiquinone system and the presence of xylose in whole-cell hydrolysates (Boekhout \& Nakase, 1998). Presently, the genus Bullera comprises approximately 30 species that belong to the Tremellales, based on various molecular phylogenetic analyses (Fell et al., 2000; Nakase, 2000; Bai et al., 2001; Scorzetti et al., 2002). Until now, two teleomorphic genera, Bulleromyces Boekhout and Fonseca (Boekhout et al., 1991) and Bulleribasidium Sampaio, Weiss and Bauer (Sampaio et al., 2002) have had

Abbreviations: ITS, internal transcribed spacer; MCMC, Markov chain Monte Carlo.

The GenBank/EMBL/DDBJ accession numbers for the sequences determined in this study are shown in Table 1. their anamorphic counterparts in Bullera. However, no teleomorph is known for most Bullera species. Implementation of molecular phylogenetic analyses based on DNA sequence data has emphasized the need to integrate mitosporic taxa, such as Bullera, into the classification system that was originally built exclusively for sexual fungi.

Ballistoconidia-producing yeasts are found frequently in the phylloplane. Forceful liberation of propagules seems to constitute an ecological advantage for colonization and dissemination in the plant leaf environment (Nakase, 2000). A 2 year survey (1997-1998) of the phylloplane yeast communities that are found on selected plants from two study sites in the Arrábida Natural Park, Portugal, was carried out by Inácio et al. (2002). This natural park encompasses different microclimatic areas with specific formations of typically Mediterranean vegetation. Among a vast majority of isolates of basidiomycetous affinity, seven yeast strains were identified preliminarily as Bullera alba Hanna, the anamorphic stage of Bulleromyces albus Boekhout and Á. Fonseca. In contrast to Bulleromyces albus, these isolates produced a brownish pigment when 
grown on potato dextrose agar. The pigment caused darkening of the cultures and diffused into the culture medium. Subsequent mating studies revealed that the new isolates did not react with the different mating types of Bulleromyces albus, but were sexually compatible with each other and produced clamped mycelium with haustoria, basidia and basidiospores in culture. Several taxonomic criteria that have been evaluated during the present study indicate that these Arrábida isolates should be accommodated in a novel genus, rather than in any existing genus of the Tremellales. Moreover, during the course of this investigation, a novel Bullera species was found among collection isolates that were identified previously as Bullera alba.

\section{METHODS}

Two methods were used for isolation: one was based on the plating of leaf washings, as described by Inácio et al. (2002), and the other on the spore-fall method. Leaves were collected from different plants (see Table 1) in the Arrábida Natural Park [for further details, see Inácio et al. (2002)]. A list of cultures of the novel taxa described in this study is shown in Table 1. For microscopy, cultures were grown on MYP agar [malt extract, $0 \cdot 7 \%(\mathrm{w} / \mathrm{v})$; yeast extract, $0 \cdot 05 \%(\mathrm{w} / \mathrm{v})$; soytone peptone, $0.25 \%(\mathrm{w} / \mathrm{v})$; and agar, $1.5 \%(\mathrm{w} / \mathrm{v})]$ at room temperature $\left(20-23^{\circ} \mathrm{C}\right)$ and examined with an Olympus BX50 microscope by using phase-contrast optics. For determination of sexual compatibility, pairs of 2-4-day-old cultures were mixed on MYP agar, incubated at room temperature and examined for production of mycelium and clamp connections after 2 weeks.

Physiological and biochemical characterization followed the methods described by Yarrow (1998). Additional assimilation tests were performed by using aldaric acids and aromatic compounds as described by Fonseca (1992) and Sampaio (1999), respectively.

For determination of the extent of DNA relatedness, total genomic DNA was extracted and purified by using the procedures described by Sampaio et al. (2001). For DNA-DNA reassociation experiments, a Gilford Response UV-VIS spectrophotometer and its thermal programming software were used and the methods of Kurtzman et al. (1980) were followed.

DNA sequence analysis, DNA extraction, PCR amplification, purification and cycle sequencing followed the protocol of Fell et al. (2000). Alignments were made with MegAlign (DNAStar) and corrected visually. To estimate phylogenetic relationships, we applied the Bayesian Markov chain Monte Carlo (MCMC) method of phylogenetic inference (Larget \& Simon, 1999), as implemented in the computer program MRBAYES (Huelsenbeck \& Ronquist, 2001). This method allows estimation of the a posteriori probability that groups of taxa are monophyletic, given the DNA alignment (i.e. the probability that corresponding bipartitions of the species set are present in the true unrooted tree, including the given species). Four incrementally heated simultaneous Monte Carlo Markov chains were run over 1000000 generations by using the general time-reversible model of DNA substitution with $\Gamma$-distributed substitution rates $(G T R+G)$, random starting trees and default starting parameters of the DNA substitution model. Trees were sampled every 100 generations, resulting in an overall sampling of 10000 trees. From those trees that were sampled after the process had reached a stationary stage, a consensus tree was computed to obtain estimates for a posteriori probabilities. This Bayesian approach to phylogenetic analysis was repeated several times, always using random starting trees and default starting values for the model parameters to test the reproducibility of the results.

\section{RESULTS AND DISCUSSION}

\section{Latin diagnosis of Auriculibuller Sampaio et Fonseca gen. nov.}

Fungus dimorphus. Basidiomata nulla. Basidia plerumque transversalitre, interdum longitudinalitre septata, fibulata, in fasciculis parvis an binatim disposita. Basidiosporae globosae, a sterigmatibus eiciuntur, gemmis vel repetitione germinant. Hyphae hyalinae, fibulatae, haustoriis tremelloideis. Status anamorphosium in genus Bulleram pertinet. Typus generis: Auriculibuller fuscus in opere ipso descriptus.

Table 1. Source, sexuality and GenBank accession numbers of cultures of the novel taxa described in this study

\begin{tabular}{|c|c|c|c|c|}
\hline Species* & Isolation source & Sexuality $\dagger$ & \multicolumn{2}{|c|}{ GenBank no. } \\
\hline PYCC $5690^{\mathrm{T}}=\mathrm{A} 2 \mathrm{AVS} 4^{\mathrm{T}}$ & Acer monspessulanum, Arrábida, Portugal & MT A1 & AF444762 & AF444668 \\
\hline PYCC $5691=$ A2AVS5 & Acer monspessulanum, Arrábida, Portugal & MT A2 & AF444763 & AF444669 \\
\hline PYCC $5739=$ A2QVSI 1 & Quercus faginea, Arrábida, Portugal & MT A2 & AF444764 & AF444670 \\
\hline 2AVF3 & Acer monspessulanum, Arrábida, Portugal & MT A2 & & \\
\hline A5QSV2 & Quercus faginea, Arrábida, Portugal & MT A2 & & \\
\hline Bullera japonica PYCC $4534^{\mathrm{T}}=\mathrm{CBS} 2013^{\mathrm{T}}$ & Zelkowa sp., Japan & ANA & AF444760 & AF444666 \\
\hline
\end{tabular}

${ }^{\star}$ CBS, Centraalbureau voor Schimmelcultures, Yeast Division, Utrecht, the Netherlands; PYCC, Portuguese Yeast Culture Collection, FCT-UNL, Portugal.

$\dagger$ ANA, Anamorphic; MT, mating type. 


\section{Description of Auriculibuller Sampaio and Fonseca gen. nov.}

Auriculibuller (Au.ri.cu.li.bul'ler. L. fem. n. auricula earlobe, referring to the auricularioid nature of the basidia; N.L. n. Bullera referring to the anamorphic yeast genus Bullera; N.L. masc. n. Auriculibuller auricularioid yeast that is similar to the genus Bullera).

Dimorphic. Basidiocarps are absent. Basidia are typically transversally septate or occasionally longitudinally septate, arranged in small clusters or in pairs and clamped. Basidiospores are globose, rotationally symmetrical, produced at the apex of tubular sterigmata and forcibly discharged; germination occurs by budding or repetition. Hyphae are hyaline and clamped with tremelloid haustoria. Anamorphic stage produces ballistoconidia and budding yeast cells and is classified in the genus Bullera. The type species is Auriculibuller fuscus.

\section{Latin diagnosis of Auriculibuller fuscus Sampaio, Inácio, Fonseca et Fell sp. nov.}

Cultura in striis post septem dies cremea, fuscans aetate progrediente. Post unum mensem cultura fusca, mucilaginea, superficie laevi, nitida. In statu unicellulari cellulae ovoideae, (3) $4-5 \times 5-8 \mu \mathrm{m}$. Sterigmata $1-2 \times 7-30 \mu \mathrm{m}$, interdum usque ad $60 \mu \mathrm{m}$ longa. Ballistoconidia globosa $(4-5 \cdot 5 \mu \mathrm{m})$ ad subglobosa $(4 \cdot 5-5 \times 6-7 \mu \mathrm{m})$. Mycelium hyphis fibulatis, $1 \cdot 5-2 \mu \mathrm{m}$ diametro, haustoriis tremelloideis. Basidia cylindracea, transversalitre septata, bicellulata. Basidiosporae globosae ad subglobosae, 5-7 $\mu \mathrm{m}$ diametro, parietibus tenuibus, eiciuntur a sterigmatibus $(1-2 \times 8-15 \mu \mathrm{m})$, gemmis vel repetitione germinant. Characteres biochemici physiologicique Auriculibulleri fusci in tabula 2 describuntur.

\section{Description of Auriculibuller fuscus Sampaio, Inácio, Fonseca and Fell sp. nov.}

Auriculibuller fuscus (fus'cus. L. masc. adj. fuscus darkcoloured, referring to the greyish-brown darkening of the cultures that occurs upon ageing).

Streak culture after 1 week is cream-coloured; becomes darker upon ageing. After 1 month, cultures are greyishbrown, mucilaginous, smooth-surfaced and glossy. Yeast cells are ovoid after 4 days on MYP agar, measuring (3) $4-5 \times 5-8 \mu \mathrm{m}$. Sterigmata are $1-2 \times 7-30 \mu \mathrm{m}$ or occasionally up to $60 \mu \mathrm{m}$ in length. Ballistoconidia are globose $(4-5 \cdot 5 \mu \mathrm{m})$ to subglobose $(4 \cdot 5-5 \times 6-7 \mu \mathrm{m})$ (Fig. 1). Hyphae are $1 \cdot 5-2 \mu \mathrm{m}$ in diameter with clamp connections and haustoria, which are formed after crossing of sexually compatible strains (Fig. 2). Haustorial cells are globose to slightly elongated (Fig. 2). Basidia initials are at first globose and then become cylindrical (Fig. 3); they are arranged in small clusters or isolated (Fig. 3). Mature basidia are two-celled, normally transversally septate and cylindrical, measuring 5-7 $\times 20-30 \mu \mathrm{m}$. Basidiospores are globose to slightly subglobose (5-7 $\mu \mathrm{m}$ in diameter), thinwalled, ejected from sterigmata that measure $1-2 \times 8-15 \mu \mathrm{m}$

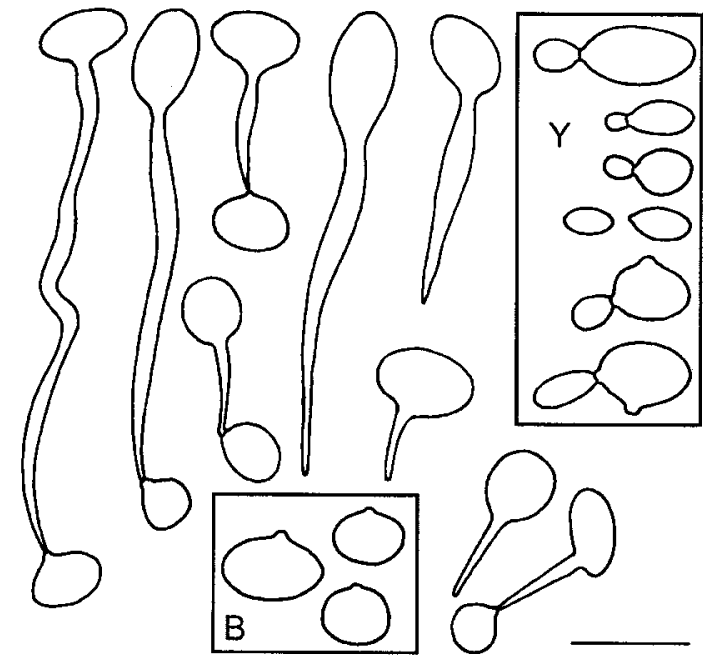

Fig. 1. Line drawings of yeast cells $(Y)$, ballistoconidiogenic cells and ballistoconidia ( $\mathrm{B}$ indicates released ballistoconidia) of Auriculibuller fuscus (PYCC $5690^{\top}$ and PYCC 5739) observed on MYP agar after 4-7 days. Bar, $10 \mu \mathrm{m}$.

and germinate by budding or repetition (Fig. 3). Biochemical and physiological properties of A. fuscus are given in Table 2 and its phylogenetic placement is shown in Fig. 5.

The type strain is PYCC $5690^{\mathrm{T}}=\mathrm{CBS} 9648^{\mathrm{T}}$. A complementary mating reference strain is PYCC $5739=$ CBS 9650. The seven cultures of A. fuscus that are listed in Table 1 were isolated in 1997 and 1998 from leaves of different Mediterranean trees and shrubs that were collected at the



Fig. 2. Line drawings of the sexual stage of Auriculibuller fuscus (PYCC $5690^{\top} \times$ PYCC 5739) observed on MYP agar after 10-15 days. Yeast cells with appressoria-like structures (upper left part), conjugation and early stages of mycelium formation (upper right part) and hyphae with clamp connections and haustoria (lower part). Bar, $10 \mu \mathrm{m}$. 


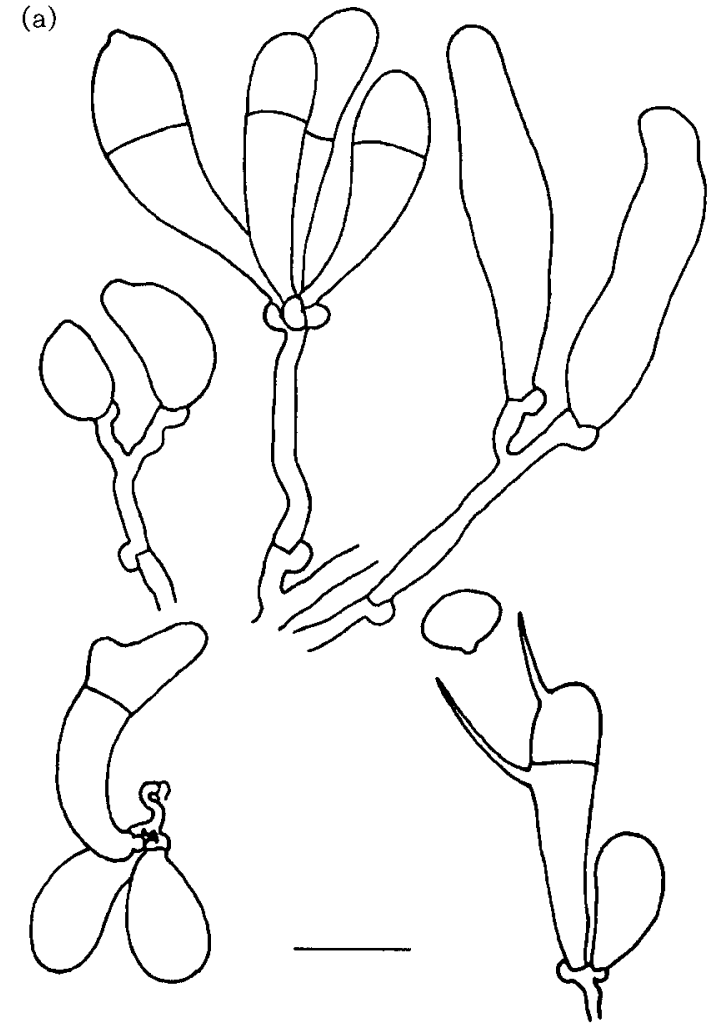

(b)

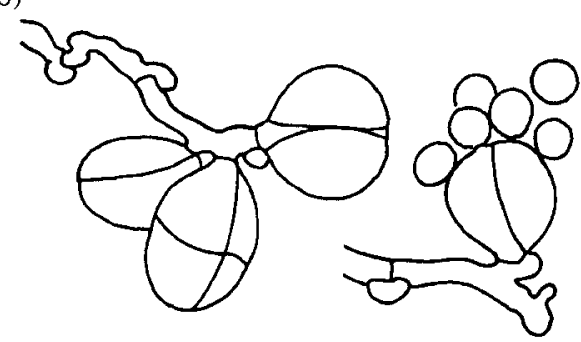

Fig. 3. Line drawings of the basidial stage of Auriculibuller fuscus (PYCC $5690^{\top} \times$ PYCC 5691) and comparison with Bulleromyces albus. (a) Basidia of $A$. fuscus in different developmental stages: non-septate globose, non-septate cylindrical, septate and septate with sterigmata; note one basidiospore between the two sterigmata on the lower right part. (b) Basidia of Bulleromyces albus (PYCC $4539 \times$ PYCC 4560); note the different basidial shape and type of septation and the sessile mode of basidiospore formation. Bar, $10 \mu \mathrm{m}$.

Arrábida Natural Park, Portugal. All cultures were isolated by using the spore-fall method, except for strain 2AVF3, which was isolated by using the leaf-washing method. Sexual compatibility and identical microsatellite-primed PCR fingerprints with primer M13 supported conspecificity of those isolates (data not shown). Sexual compatibility seems to be biallelic, as crossing experiments suggest the presence of two mating types.

A. fuscus is able to complete its life cycle on culture media. When sexually compatible strains are mixed on MYP agar,
Table 2. Physiological and biochemical characteristics of Auriculibuller fuscus and Bullera japonica

Taxa: 1, Auriculibuller fuscus; 2, Bullera japonica. Characteristics are scored as: +, growth; -, no growth; D, delayed growth; $\mathrm{V}$, variable growth. Both taxa were positive for assimilation of the carbon compounds D-glucose, D-galactose, D-glucosamine, D-ribose, D-xylose, L-arabinose, D-arabinose, L-rhamnose, sucrose, maltose, $\alpha, \alpha$-trehalose, methyl $\alpha$-D-glucoside, cellobiose, salicin, melibiose, lactose, raffinose, melezitose, soluble starch, erythritol, ribitol, xylitol, D-glucitol, D-mannitol, inositol, glucono- $\delta$-lactone, D-gluconic acid, D-glucuronic acid, succinic acid, citric acid, Lmalic acid, saccharic acid, mucic acid and gallic acid and the nitrogen compounds ethylamine, L-lysine and cadaverine. Both taxa were positive for growth with $0.01 \%$ cycloheximide, growth at $25^{\circ} \mathrm{C}$, formation of starch-like compounds, splitting of arbutin, hydrolysis of urea and the diazonium blue $\mathrm{B}$ reaction. Both taxa were negative for fermentation of D-glucose, assimilation of the carbon compounds inulin, D-tartaric acid, methanol, ethanol, vanillic acid, ferulic acid, veratric acid, $p$-hydroxybenzoic acid, $m$ hydroxybenzoic acid, protocatechuic acid, catechol, salicylic acid, gentisic acid and phenol and the nitrogen compounds potassium nitrate, sodium nitrite, creatine and creatinine. Both taxa were negative for growth in vitamin-free medium, growth with $0 \cdot 1 \%$ cycloheximide and growth at $35{ }^{\circ} \mathrm{C}$ (these data are available in tabular form at http://www.crem.fct.unl.pt/dimorphic_basidiomycetes/ Databases/databases.htm).

\begin{tabular}{|lcc|}
\hline Characteristic & $\mathbf{1}$ & $\mathbf{2}$ \\
\hline Carbon compounds: & & \\
L-Sorbose & - & $\mathrm{D}$ \\
Glycerol & $\mathrm{V}$ & $\mathrm{D}$ \\
Galactitol & $\mathrm{V}$ & + \\
DL-Lactic acid & $\mathrm{V}$ & + \\
L-Tartaric acid & $\mathrm{V}$ & + \\
D-Tartaric acid & $\mathrm{V}$ & + \\
Growth at $30^{\circ} \mathrm{C}$ & $\mathrm{V}$ & + \\
\hline
\end{tabular}

cells with appressorium-like structures develop (Fig. 2). Conjugation between two cells is difficult to observe, due to the twisting and ramifications of the conjugating hyphae (Fig. 2). One-week-old crossings show conspicuous clamped mycelium with haustoria that are able to attach to hyphae of deuteromycetous culture contaminants, which suggests that A. fuscus is capable of mycoparasitism in nature. Basidia initials are formed after 6-8 weeks at room temperature. Transfer of agar blocks of this age to $2 \%$ water agar stimulated basidial formation. Mature basidia (septated, with sterigmata and basidiospores) could be detected approximately 1 week after transfer to water agar.

\section{Latin diagnosis of Bullera japonica Sampaio, Fonseca et Fell sp. nov.}

Cultura in striis post septem dies cremea, fuscans aetate progrediente. Post unum mensem cultura fusca, mucilaginea, superficie laevi, nitida. Cellulae ovoideae ad cylindraceae, 
(1.5) $\quad 2-3 \times 5-10 \quad(12) \mu \mathrm{m}$. Sterigmata $1-2 \times 8-16 \mu \mathrm{m}$. Ballistoconidia globosa (5-6 $\mu \mathrm{m}$ diametro) ad subglobosa $(4-5 \times 5-6 \mu \mathrm{m})$. Characteres biochemici physiologicique Bullerae japonicae in tabula 2 describuntur.

\section{Description of Bullera japonica Sampaio, Fonseca and Fell sp. nov.}

Bullera japonica [ja.po'ni.ca. N.L. adj. japonica referring to the origin (Japan) of the single culture of this species that is presently known].

Streak culture after 1 week is cream-coloured; becomes darker upon ageing. After 1 month, cultures are greyishbrown, mucilaginous, smooth-surfaced and glossy. Yeast cells are ovoid to cylindrical after 4 days on MYP agar, measuring $(1 \cdot 5) \quad 2-3 \times 5-10 \quad(12) \mu \mathrm{m}$. Sterigmata are $1-2 \times 8-16 \mu \mathrm{m}$; ballistoconidia are globose $(5-6 \mu \mathrm{m})$ to subglobose $(4-5 \times 5-6 \mu \mathrm{m})$ (Fig. 4). Biochemical and physiological properties of Bullera japonica are presented in Table 2 and its phylogenetic placement is shown in Fig. 5.

The type strain of Bullera japonica (PYCC $4534^{\mathrm{T}}=\mathrm{CBS}$ $2013^{\mathrm{T}}$ ) was isolated by $\mathrm{K}$. Tubaki in Japan from Zelkowa sp.

The phylogenetic placement of A. fuscus and Bullera japonica was inferred by using the D1/D2 domains of the 26S rDNA and the Bayesian MCMC method of phylogenetic inference (Fig. 5). As expected, both taxa belong to the Tremellales. Sequence data indicated that the two novel taxa described in this report are related most closely to one another. Their D1/D2 sequences differed by three nucleotide substitutions and one single-base indel. The four strains of A. fuscus that were sequenced (see Table 1) have identical sequences; therefore, only the sequence of the type strain was used in molecular analyses. In the analysis shown in Fig. 5, the pair A. fuscus-Bullera japonica has, as its closest teleomorphic relative, Papiliotrema bandonii Sampaio, Gadanho, Weiss and Bauer. Both Auriculibuller and Papiliotrema have auricularioid basidia. In order to study in more detail the relationship between

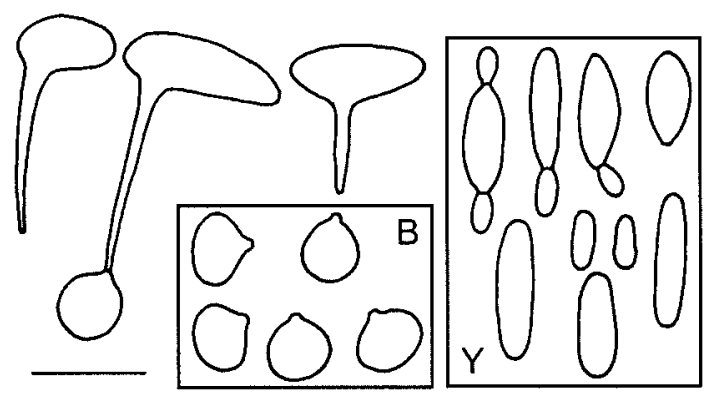

Fig. 4. Line drawings of yeast cells $(Y)$, ballistoconidiogenic cells and ballistoconidia ( $\mathrm{B}$ indicates released ballistoconidia) of Bullera japonica (PYCC $4534^{\top}$ ) observed on MYP agar after 4-7 days. Bar, $10 \mu \mathrm{m}$.
A. fuscus and Bullera japonica, the complete internal transcribed spacer (ITS) region (ITS1 $+5 \cdot 8 \mathrm{~S}$ rDNA + ITS2) of the two taxa was investigated (the phylogenetic tree is available at http://www.crem.fct.unl.pt/dimorphic_ basidiomycetes/Databases/databases.htm). Again, the four strains of $A$. fuscus showed identical sequences that differed from that of Bullera japonica by seven substitutions and one single-base indel. In ITS analysis, $P$. bandonii was the closest relative of $A$. fuscus and Bullera japonica. Nuclear DNA-DNA reassociation experiments between two strains of A. fuscus (PYCC $5690^{\mathrm{T}}$ and PYCC 5739) yielded high relatedness values (92-93\%), whereas each of these strains produced intermediate values in reassociation experiments with Bullera japonica (52-58\% relatedness). Moreover, crossings of Bullera japonica with the two mating types of A. fuscus invariably gave negative results. Physiological and biochemical characterization of the two novel taxa, presented in Table 2, showed that, except for the assimilation of L-sorbose, A. fuscus and Bullera japonica are indistinguishable. As indicated in Table 2, a few tests gave variable results for $A$. fuscus.

The two novel taxa share a peculiar cultural characteristic: when grown on potato dextrose agar, they produce a brownish pigment that darkens the cultures and diffuses into the agar. This trait is absent in Bulleromyces albus and motivated a deeper analysis of the pigment-producing isolates.

The genus Auriculibuller, described in the present report, is the third genus to accommodate the sexual stages of the mitosporic ballistoconidial genus Bullera. The other two genera are Bulleromyces and the recently described genus Bulleribasidium (Sampaio et al., 2002). Despite similar basidial morphologies, Auriculibuller and Bulleribasidium are clearly distinct, based on the molecular phylogenetic hypothesis presented in Fig. 5. From an evolutionary point of view, Auriculibuller and Bulleromyces seem to share a recent common ancestor. The phylogenetic placement of these two genera correlates with the morphological comparison that is also presented in Fig. 5. Whereas A. fuscus, $P$. bandonii and Tremella exigua Desm. have clavate to cylindrical basidia with obliquely to transverse septa, Bulleromyces albus and three Tremella species of the Indecorata group sensu Chen 1998, namely Tremella indecorata Sommerf. Fr., Tremella moriformis (Fr.) Smith ex Berk. and Tremella nivalis C.-J. Chen, have subglobose to pyriform, longitudinally or obliquely cruciate-septate basidia. In Fig. 5, the clade for which basidial types are presented contains two well-supported monophyletic groups. One includes the species with a tremelloid basidial plan and the other includes Auriculibuller and Papiliotrema, which have clavate basidia with obliquely to transverse septation. This topology was also observed in maximumparsimony analysis (data not shown), but the bootstrap values were generally lower that the a posteriori probability values of MCMC analyses. As can be observed in the illustrations that are included in Fig. 5, T. exigua shows 


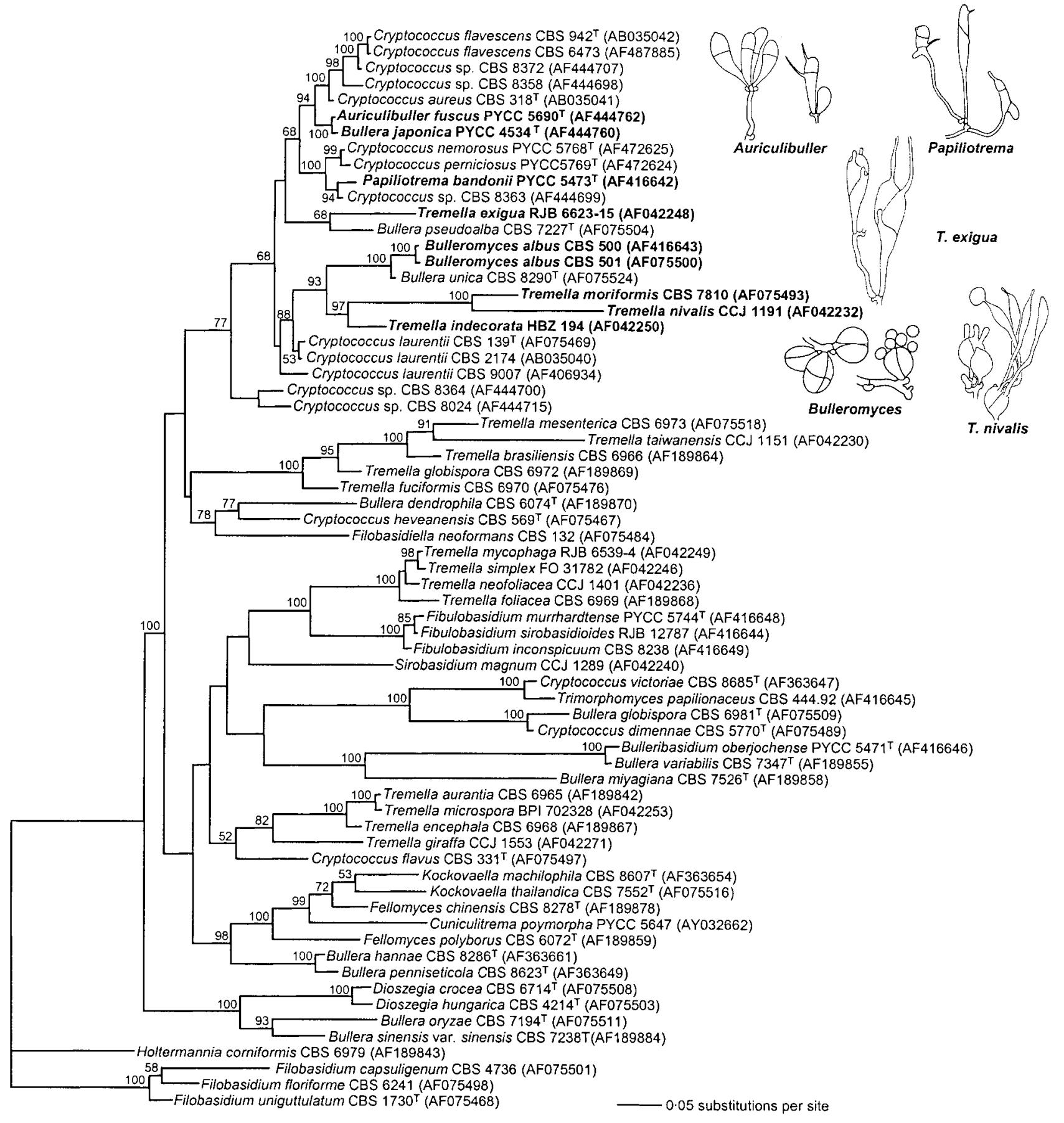

Fig. 5. Phylogenetic tree of Auriculibuller fuscus, Bullera japonica and related taxa of the Tremellales, inferred by using Bayesian MCMC analysis of an alignment of the D1/D2 region of $26 \mathrm{~S}$ rDNA. Numbers on branches are estimates for a posteriori probabilities, i.e. probabilities that the respective groups are monophyletic given the alignment. The topology was rooted with Filobasidium floriforme, Filobasidium uniguttulatum and Filobasidium capsuligenum. Names in bold type correspond to organisms discussed in the comparison of basidial types and/or to novel taxa described in this report. GenBank accession numbers of sequences are indicated after strain numbers. Schematic illustrations of basidial types are inserted for Auriculibuller, Bulleromyces, Papiliotrema [redrawn from Sampaio et al. (2002)] and selected Tremella species [redrawn from Chen (1998); the basidial types of Tremella moriformis and Tremella indecorata are basically similar to that of Tremella nivalis and are not shown]. 
an intermediate basidial morphology. In the various MCMC analyses that were carried out during this study, the position of this species was found to vary and never received high statistical support. In the ITS tree (see Supplementary Figure, available at http://www.crem.fct.unl. pt/dimorphic_basidiomycetes/Databases/databases.htm), species with Tremella-type basidia were again well-separated from Auriculibuller and Papiliotrema, the latter being placed in the vicinity of the novel genus that is described in this report. The possible conspecificity between A. fuscus and Bullera japonica, which is suggested by pigment production, nutritional similarity and D1/D2 sequence data, was ruled out, based on greater sequence divergence of ITS data, absence of sexual compatibility and lack of high DNADNA reassociation values.

The unravelling of novel sexual taxa and mitosporic species, such as those described here, is essential to expand our assessment of the diversity of the Tremellales and allied taxa of the Tremellomycetidae. Accumulation of more data on the Tremellales by means of multidisciplinary approaches will set the basis for a more accurate perspective on the evolutionary history of these organisms and will consequently contribute to improved classification proposals. Another issue in the systematics of the Tremellales is the genus concept for this group. The polyphyletic nature of Tremella, as depicted in Fig. 5, suggests that the tremelloid basidial type, the key character for the genus, needs to be combined with other markers for adequate generic differentiation. Molecular studies have also indicated that asexual genera, such as Bullera and Cryptococcus Vuillemin, are polyphyletic (Fell et al., 2000). On the other hand, the same approaches have validated the generic concept of Fibulobasidium Bandoni and fostered the segregation of species from heterogeneous assemblages into natural groups, such as Dioszegia Zsolt emend. Takashima, Deak and Nakase (Takashima et al., 2001). The proposal of Auriculibuller in this report follows this trend.

\section{ACKNOWLEDGEMENTS}

J. I. and M. G. were supported by grants PRAXIS/XXI/BD/19833 and SFRH/BD/1170/2000, respectively, and J. W. F. was supported by the USA National Science Foundation, Division of Environmental Biology (DEB 0206521). We thank Michael Weiß (University of Tübingen, Germany) for preparing the Latin diagnoses and for advice concerning the MCMC phylogenetic analyses.

\section{REFERENCES}

Bai, F.-Y., Takashima, M. \& Nakase, T. (2001). Phylogenetic analysis of strains originally assigned to Bullera variabilis. descriptions of Bullera pseudohuiaensis sp. nov., Bullera komagatae sp. nov. and Bullera pseudoschimicola sp. nov. Int J Syst Evol Microbiol 51, 2177-2187.
Boekhout, T. \& Nakase, T. (1998). Bullera Derx. In The Yeasts, a Taxonomic Study, 4th edn, pp. 731-741. Edited by C. P. Kurtzman \& J. W. Fell. Amsterdam: Elsevier.

Boekhout, T., Fonseca, A. \& Batenburg-van der Vegte, W. H. (1991). Bulleromyces genus novum (Tremellales), a teleomorph for Bullera alba, and the occurrence of mating in Bullera variabilis. Antonie van Leeuwenhoek 59, 81-93.

Chen, C.-J. (1998). Morphological and molecular studies in the genus Tremella. Bibl Mycol 174, 1-225.

Fell, J. W., Boekhout, T., Fonseca, A., Scorzetti, G. \& StatzellTallman, A. (2000). Biodiversity and systematics of basidiomycetous yeasts as determined by large-subunit rDNA D1/D2 domain sequence analysis. Int J Syst Evol Microbiol 50, 1351-1371.

Fonseca, A. (1992). Utilization of tartaric acid and related compounds by yeasts: taxonomic implications. Can J Microbiol 38, 1242-1251.

Huelsenbeck, J. P. \& Ronquist, F. (2001). MRBAYES: Bayesian inference of phylogenetic trees. Bioinformatics 17, 754-755.

Inácio, J., Pereira, P., Carvalho, M., Fonseca, Á., Amaral-Collaço, M. T. \& Spencer-Martins, I. (2002). Estimation and diversity of phylloplane mycobiota on selected plants in a Mediterranean-type ecosystem in Portugal. Microbial Ecol 44, 344-353.

Kurtzman, C. P., Smiley, M. J., Johnson, C. J., Wickerham, L. J. \& Fuson, G. B. (1980). Two new and closely related heterothallic species, Pichia amylophila and Pichia mississippiensis: characterization by hybridization and deoxyribonucleic acid reassociation. Int J Syst Bacteriol 30, 208-216.

Larget, B. \& Simon, D. L. (1999). Markov chain Monte Carlo algorithms for the Bayesian analysis of phylogenetic trees. Mol Biol Evol 16, 750-759.

Nakase, T. (2000). Expanding world of ballistosporous yeasts: distribution in the phyllosphere, systematics and phylogeny. J Gen Appl Microbiol 46, 189-216.

Sampaio, J. P. (1999). Utilization of low molecular weight aromatic compounds by heterobasidiomycetous yeasts: taxonomic implications. Can J Microbiol 45, 491-512.

Sampaio, J. P., Gadanho, M., Santos, S., Duarte, F. L., Pais, C., Fonseca, A. \& Fell, J. W. (2001). Polyphasic taxonomy of the basidiomycetous yeast genus Rhodosporidium: Rhodosporidium kratochvilovae and related anamorphic species. Int J Syst Evol Microbiol 51, 687-697.

Sampaio, J. P., Weiß, M., Gadanho, M. \& Bauer, R. (2002). New taxa in the Tremellales: Bulleribasidium oberjochense gen. et $\mathrm{sp}$. nov., Papiliotrema bandonii gen. et sp. nov. and Fibulobasidium murrhardtense sp. nov. Mycologia 94, 873-887.

Scorzetti, G., Fell, J. W., Fonseca, A. \& Statzell-Tallman, A. (2002). Systematics of basidiomycetous yeasts: a comparison of large subunit D1/D2 and internal transcribed spacer rDNA regions. FEMS Yeast Res 2, 495-517.

Takashima, M., Deak, T. \& Nakase, T. (2001). Emendation of Dioszegia with redescription of Dioszegia hungarica and two new combinations, Dioszegia aurantiaca and Dioszegia crocea. J Gen Appl Microbiol 47, 75-84.

Yarrow, D. (1998). Methods for the isolation, maintenance and identification of yeasts. In The Yeasts, a Taxonomic Study, 4th edn, pp. 77-100. Edited by C. P. Kurtzman \& J. W. Fell. Amsterdam: Elsevier. 